\title{
Phenotype Classification of Zebrafish Embryos by Supervised Learning
}

\author{
Nathalie Jeanray ${ }^{1}$, Raphaël Marée ${ }^{2,3}$, Benoist Pruvot ${ }^{1}$, Olivier Stern², Pierre Geurts ${ }^{2}$, Louis \\ Wehenkel $^{2}$, Marc Muller ${ }^{1}$
}

\begin{abstract}
Address: ${ }^{1}$ GIGA-Development, Stem cells and regenerative medicine, Molecular Biology and Genetic Engineering, University of Liège, ${ }^{2}$ GIGA-Systems Biology and Chemical Biology, Dept. EE \& CS, University of Liège and ${ }^{3}$ GIGA Bioinformatics Core Facility, University of Liège.
\end{abstract}

Keywords: Phenotype classification, supervised learning, zebrafish

\begin{abstract}
Zebrafish is increasingly used to assess biological properties of chemical substances and thus becomes a specific tool for toxicological and pharmacological studies. The effects of chemical substances on embryo development are generally evaluated manually through microscopic observation by an expert and documented by several typical photographs. Ideally, all the evaluated individuals should be photographed and classified according to the defects observed. Our project aims at reducing the workload and time required for the biological expert by automatic data acquisition through motorized microscopy, followed by classification of the obtained images. In order to increase the reproducibility and the objectivity of this classification, we present here a method to classify images of zebrafish embryos according to their defects automatically using a supervised learning approach. Automation of the analysis and classification of zebrafish pictures would become a real advantage for the biologists in terms of time and accuracy.
\end{abstract}

\section{Introduction}

Zebrafish, or "Danio rerio", is commonly used as a vertebrate model organism in the fields of develop ${ }^{1}$ mental biology, but also increasingly in toxicology and pharmacology. Due to several advantages such as fast growth, ex vivo development, larvae transparency, low cost and permeability to small molecules, zebrafish appears as a powerful model to assess toxic activities on vertebrates. Typically, embryos are collected one by one and observed manually in order to detect potential developmental or

Appearing in Proceedings of the 20th Machine Learning conference of Belgium and The Netherlands. Copyright 2011 by the author(s)/owner(s). morphological modifications. These modifications are listed, illustrated by a picture and statistically processed to infer the toxicological effects of the drug and the role it might play within the metabolic reactions. This method is tedious, time-consuming and prone to appreciation subjectivity. The large number of substances to be tested and the need for accuracy of the results call for methods allowing automation of both data acquisition and classification of the images.

\section{Methods}

\subsection{Treatments}

Zebrafish embryos were treated at 2 days post fertilization in batches of 25 individuals and analyzed after 24 hours of treatment. At this stage (3 days old), the embryos have hatched and are easily observable. Untreated control batches received only the solvent used for the drug stock solution.

\subsection{Data Acquisition}

In order to develop an accurate and non-biased approach to classify the pictures, a particular attention must be given to image acquisition. Photographs have been taken on an Olympus stereo dissecting microscope with the same parameters from one acquisition to the next (exposure time $=10 \mathrm{~ms}$, contrast $=1.05$, maximum luminosity, white balance, magnification $=1.60 \mathrm{x}$ ) to limit bias errors due to a non homogenous background. Embryos are placed in a melt of E3 and methylcellulose in a 12-well plate, one fish per well. The pictures are taken manually at this stage of the work.

\subsection{Automated recognition pipeline}

\subsubsection{IMAGE PRE-PROCESSING}

Embryo images are first standardized based on three major steps. First, we apply a rotation in order to place all the embryos in the same position, which is, horizontally and head to the left. This step is performed automatically by an algorithm searching the position of the eyes and rotating the fish when needed. Secondly, the dorso-ventral orientation of the fish is determined 


\section{Phenotype Classification of Zebrafish Embryos by Supervised Learning}

by a supervised classifier trained with dorsal and ventral examples, then we applied a flipping operation if needed. The third step consists of cropping the fish using connected component labeling.

Further pre-processing steps have been evaluated depending on the sought defect. In the case of pericardial edemas, we tested automatic cropping of the fish to keep only the anterior ventral part. For the curved tail, we applied an algorithm to cut off the head of the fish to concentrate on the trunk of the fish.

\subsubsection{IMAGE LABELING}

After standardization, images of embryos were labeled by three biologists working independently. The goal is to identify two types of malformations on embryos, and unaffected ones. The embryos observed have been treated with a medication at different concentrations. Most of these embryos developed some abnormal phenotypes as, for instance, pericardial edemas, curved tails and growth delay. We chose to focus our analyses on two deformations at first, pericardial edema and curved tail. Examples are shown in Figure 1.

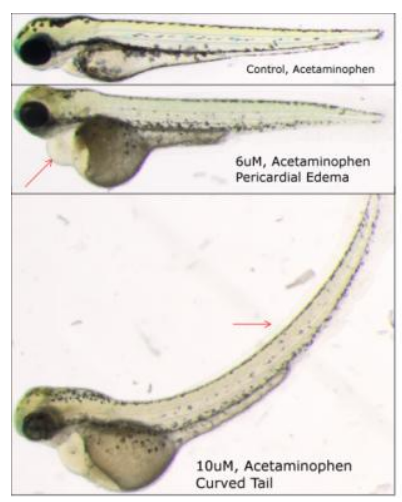

Figure 1. Illustration of the phenotypes analyzed in this study. Red arrows indicate the phenotypes studied. The top picture is a normal fish, the middle one presents a pericardial edema while the bottom image shows a curved tail.

\subsubsection{SUPERVISED IMAGE CLASSIFICATION}

Given the set of training images where each image was labeled into the majority class assigned by the three experts, the goal is to build a model by supervised learning that will be able to predict accurately the class of new, unseen images. We used the image classification algorithm of (Marée et al., 2007), a generic method which has been validated on many problems before envisaging the development of a more specific method. It is based on dense random subwindow extraction in images, their description by raw pixel values, and the use of ensembles of extremely randomized trees (Geurts et al., 2006) to classify these subwindows hence images.

\section{Results}

We evaluated our method on images acquired in three independent experiments with different numbers of images per class for each experiment (Figure 2).

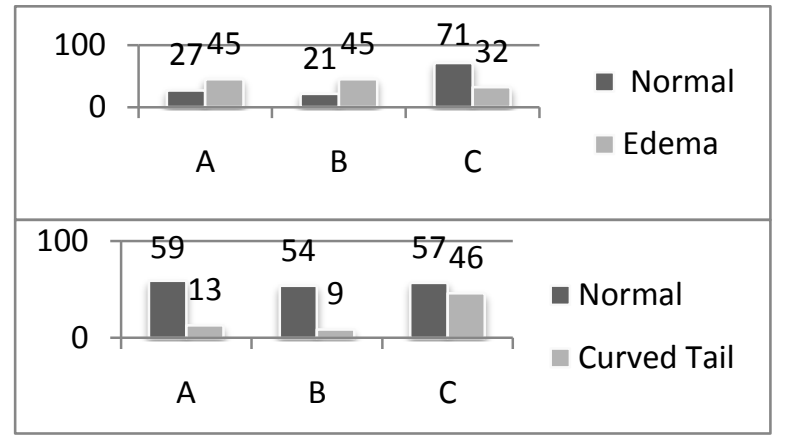

Figure 2. Number of images in each class (normal, edema or curved tail) in the three experiments A, B and C.

For each experiment and each deformation, we evaluate empirically the influence of the main parameters of the algorithm by cross-validation or leave-one-out protocols. Table 1 shows the best recognition rates we obtained. The parameters we modified in order to get these results were mainly the size ranges of the extracted subwindows, the classification scheme, the number of trees to build, the stop criterion based on the minimum node sample size, the number of random tests and the number of subwindows extracted within each image. Binary models are then built since we try to classify larvae presenting an edema vs. control larvae (edema vs. normal), and larvae with a curved tail vs. normal ones (curved tail vs. normal).

Table 1. Classification accuracies for "Edema" and "Curved Tail" classes.

\begin{tabular}{lccc}
\hline DATA SET & A & B & C \\
\hline EDEMA & 96.0 & 59.9 & 98.4 \\
CURVED TAIL & 87.5 & 75.8 & 94.9 \\
\hline
\end{tabular}

\section{Conclusion and Perspectives}

Our automatic classification method already gives promising results in the analysis of two different defects, edema and curved tails, allowing to anticipate that other morphological abnormalities could also be classified. In the future, we will focus on rendering the acquisition procedure fully automatic, while also extending our classification method to other defects.

\section{References}

Marée, R., Geurts, P., Wehenkel, L. (2007). Random subwindows and extremely randomized trees for images classification in cell biology. BMC Cell Biology, 8:S2

Geurts P, Ernst D, Wehenkel L. (2006). Extremely Randomized Trees. Machine Learning, 36:3-42 